\title{
Novel candidate genes for grain texture in Russian wheat varieties
}

\author{
Kiseleva A.A. ${ }^{1 *}$, Leonova I.N. ${ }^{2}$, Pshenichnikova T.A. ${ }^{2}$, Salina E.A. ${ }^{1}$ \\ ${ }^{1}$ Kurchatov Genomics Center, Institute of Cytology and Genetics SB RAS, Novosibirsk, Russia \\ ${ }^{2}$ Institute of Cytology and Genetics SB RAS, Novosibirsk, Russia \\ *e-mail: antkiseleva@bionet.nsc.ru
}

Key words: Triticum aestivum, grain texture, grain hardness, puroindolines, GWAS

Motivation and Aim: Grain texture is an important milling characteristic of common wheat. Previously, it was shown that the Ha locus on 5DS, containing the puroindoline a, puroindoline $b$ and GSP-1 genes, has a crucial role in endosperm texture. Puroindoline-like genes mapped to chromosome group 7 also contribute to this trait. However, some other loci were detected on different chromosomes, but no specific genes were proposed to be involved in grain hardness organization.

Methods and Algorithms: There were 92 varieties adapted to the environments of the Siberia region used in this study. We estimated two parameters for grain texture in two years: flour particle diameter (FPD, $\mu \mathrm{m})$ and flour particle specific surface (FPSS, $\mathrm{cm} 2 / \mathrm{g}$ ). SNP genotyping was performed using the Illumina Infinium $15 \mathrm{k}$ Wheat platform by TraitGenetics $\mathrm{GmbH}$. The population structure was analysed using the R-based STRUCTURE-like inference algorithm LEA. For association analysis, a mixed model approach implemented in the R package "GENESIS" was used. The resulting mixed linear model considered fixed effects of SNPs and population structure and random effects for kinship. To estimate the expression of genes in the detected loci, we used data of the common wheat cultivar Azhurnaya developmental time course.

Results: Individual variance component analysis demonstrated that the genotypic variance principally contributed to flour particle size (79\%) and flour particle-specific surface (80\%). The assumption of ancestral populations $(\mathrm{K})$ with cross-entropy demonstrated that the most likely number of subpopulations was $\mathrm{k}=4$. The genotyping with gene-specific primers demonstrated that twenty-three varieties had allele Pina-DIk, which lacked both Pina and Pinb genes (double null allele). Association analysis revealed a total of 26 SNPs and Pina gene-specific marker to be significantly associated with grain texture. The most promising were SNPs on chromosomes $5 \mathrm{~B}$ and 7B. These MTAs demonstrated the highest significance after FDR correction. We selected four genes located on 5B (TraesCS5B02G010300, TraesCS5B02G010400, TraesCS5B02G010800, and TraesCS5B02G011700) highly expressed in grain at the hard dough and ripening stages and in the embryo proper.

Conclusion: In this study, we confirmed the crucial role of puroindoline genes in grain texture determination of Russian spring wheat varieties. We have demonstrated that rare allele Pina-Dlk is widely distributed in the studied panel of accessions. Likewise, we found a number of novel loci to be highly associated with grain texture and dissected genes from these loci. These genes are involved in the metabolism of galactolipids (DGDG) and carbohydrates (1,3- $\beta$-glucan).

Acknowledgements: The study was supported by Kurchatov Genomics Center of ICG (075-15-2019-1662). 